\title{
Concurrent Chemoradiation for T3 Glottic Squamous Cell Carcinoma: A Reasonable Alternative to Upfront Laryngectomy
}

\author{
Charles $\operatorname{Lin}^{1}{ }^{*}$, Edward $\operatorname{Sia}^{2}$ and Jacqui Keller ${ }^{1}$ \\ ${ }^{1}$ Cancer Care Services, Royal Brisbane and Women's Hospital, Herston, Australia \\ ${ }^{2}$ Queensland Health, Nambour General Hospital, Nambour, Australia
}

\begin{abstract}
Introduction

Upfront treatment options for stage III and IV larynx Squamous Cell Carcinoma (SCC) include laryngectomy or chemoradiation (CRT). The functional consequence of laryngectomy is debilitating (instant loss of voice in most cases) and significantly affects patients' quality of life. This study examined the rate of organ preservation and associated late morbidity for T3N+/-M0 glottic SCC treated with upfront concurrent CRT.
\end{abstract}

\section{Methods}

Between January 2000 and November 2007, 17 patients presented with T3N+/-M0 glottic SCC and were treated with definitive concurrent CRT at the Royal Brisbane and Women's Hospital, Australia. The primary end point was laryngectomy free survival at 5 years (5Yr LFS). The secondary end points were 5 years local control ( $5 Y$ r LC), relapse free survival (5Yr RFS), disease specific survival (5Yr DSS) and overall survival (5Yr OS). Prevalence of $\geq$ grade 2 voice changes and dysphagia were also reported.

\section{Results}

The rate of 5 Yr LFS, 5 Yr RFS, 5 Yr LC, 5 Yr DSS and 5 Yr OS were $81 \%, 71 \%, 87 \%, 89 \%$, and $89 \%$ respectively, The prevalence of $\geq$ grade 2 voice changes at 12 months post-treatment and date last seen were $44 \%$ and $33 \%$ respectively. The prevalence of $\geq$ grade 2 dysphagia at 12 months post-treatment and date last seen were $6 \%$ and $6 \%$ respectively.

\section{Conclusions}

Definitive concurrent CRT is a reasonable alternative to upfront laryngectomy for patients with T3N+/-M0 glottic carcinoma without significant morbidities.

Keywords: Organ Preservation; Concurrent Chemoradiation; Glottis; Squamous Cell Carcinoma.

*These authors contributed equally to this work

Copyright (C) 2012 Charles Lin, Edward Sia and Jacqui Keller. This is an open access article distributed under the Creative Commons Attribution License unported 3.0, which permits unrestricted use, distribution, and reproduction in any medium, provided that original work is properly cited. Contact author: Charles Lin E-mail: Charles_Lin@health.qld.gov.au 


\section{Introduction}

Upfront treatment options for stage III and IV larynx carcinoma include laryngectomy and chemoradiation (CRT). While upfront surgery +/- adjuvant radiotherapy (RT) can achieve excellent locoregional control, the functional consequence of laryngectomy can be debilitating (instant loss of voice in most cases). Previous studies comparing the two treatment approaches reported 40\%-50\% rate of larynx preservation with upfront chemoradiation (1991, Forastiere AA, 2003) while overall survival was similar.

On the other hand, long term late dysphagia associated with definitive CRT for local and/or regional advanced larynx SCC can significantly impact on patients' daily function. Incompetent larynx can occur after chemoradiation which often requires a laryngectomy to protect patients' airway. In our combined head and neck clinic at the Royal Brisbane and Women's Hospital, head and neck surgeons frequently recommend upfront laryngectomy for T3 glottic squamous cell carcinoma (SCC) due to concerns of long term dysphagia and laryngeal incompetence if patients were to receive upfront CRT. On the other hand, radiation oncologists often like to provide patients with the option of larynx preservation for this group of patients. There are fewer debates for T4 glottic SCC as the rate of organ preservation is lower than $\mathrm{T} 3$ disease and these patients are generally managed with upfront laryngectomy followed by adjuvant RT or CRT. To resolve the constant division in opinion for management of T3 glottic SCC, we conducted an audit of patients treated with upfront CRT in our institution. The rate of successful organ preservation and associated late morbidities associated with upfront concurrent CRT were reported in this paper.

\section{Methods}

Between May 2000 and November 2007, 17 patients at the Royal Brisbane and Women's Hospital received upfront CRT for T3N-/+M0 glottic SCC with curative intent. All of these patients had biopsy proven SCC of the glottis and were staged with CT scans of the head and neck and panendoscopy as a minimum.
Tumours originated from other larynx subsites were excluded. Conventional two dimensional radiotherapy technique was employed for the first 15 (88\%) patients. Three dimensional conformal radiotherapy utilizing CT planning was used for the last 2 $(12 \%)$ patients.

A retrospective chart review was conducted and relevant data collected. Severity of late voice changes and dysphagia were graded using the Common Toxicity Criteria (CTC), version 3 (Insitutute, 2006). Voice changes and dysphagia were collected at baseline prior to CRT, 12 months (+/- 2 months) after completion of CRT, and at date last seen. Treatment details, disease and survival status were also obtained. Pattern of failure was described for patients who developed recurrences. The study had approval from the local Human Resource Ethics Committee.

Voice changes and degree of dysphagia at baseline were graded based upon the initial description in the chart made by the clinicians. After completion of CRT, voice changes and dysphagia were assessed by the clinicians on patients who were alive, free of disease, and not had laryngectomy.

\section{Statistics}

The primary end point was laryngectomy free survival at 5 year (5Yr LFS). Events included for the primary end point were laryngectomy after completion of CRT and death due to any cause. Secondary end points were disease specific survival at 5year, relapse free survival at 5 year and overall survival at 5 year (5Yr DSS, 5 Yr RFS and $5 \mathrm{Yr}$ OS). The rates of all the primary and secondary end points were calculated actuarially using Kaplan-Meier product limit method.

The prevalence of $\geq$ grade 2 voice changes and dysphagia at baseline, 12 months $(+/-2$ months) after CRT, and at date last seen were illustrated using bar graphs.

\section{Results}

The median age of the cohort was 64 years (42-77). The duration of median follow up was 41 months (10-108). There were 15 (88\%) males and 2 (12\%) females. Sixteen 
patients (94\%) had N0 disease while 1 patient $(6 \%)$ had N1 disease. No patients presented with N2 or N3 diseases. Nine patients presented with fixed vocal cord (53\%). Twelve patients (71\%) had paraglottic extension. The median dose of radiotherapy received was 70Gy (62-72.5). Radiotherapy was delivered at 2-2.07Gy per fraction at 5 fractions per week. Cervical lymph nodes were irradiated in 13 patients (76\%). All patients received platinum-based chemotherapy. High dose three weekly Cisplatin (100mg per meter square) was administered to 10 patients (59\%). Weekly lower dose Cisplatin (40mg per meter square) was received by 6 patients $(35 \%)$ while one patient $(6 \%)$ received Cisplatin and 5-Fluouracil.

In terms of radiotherapy compliance, all but 2 patients completed the prescribed dose of RT. One patient received 66Gy out of the intended 70Gy due to complex social circumstances. The other patient developed severe mucosal infection and delirium. The total dose to the larynx was thus reduced to
62Gy in 33 fractions. From the chemotherapy perspective, 2 patients failed to receive the full prescribed regimen. One patient received only one out of the intended 7 cycles of weekly Cisplatin due to deteriorating renal function. The other patient received 2 instead of the prescribed 3 cycles of high dose three weekly Cisplatin due to neutropenia.

Out of the entire cohort, 4 patients recurred. One patient developed local recurrence only and was successfully salvaged with total laryngectomy. One patient developed nodal recurrence (did not receive elective nodal irradiation) and was salvaged with neck dissection without receiving further radiotherapy. One patient developed distant metastatic disease and subsequently died. One patient developed both local and regional recurrences (did receive nodal RT) but refused salvage surgery and was subsequently lost to follow up. The rate of 5Yr LFS, 5Yr RFS, 5Yr DSS and 5Yr OS were $81 \%, 71 \%, 89 \%$ and $89 \%$ respectively. (Figure1).

\section{T3 Glottic SCC}

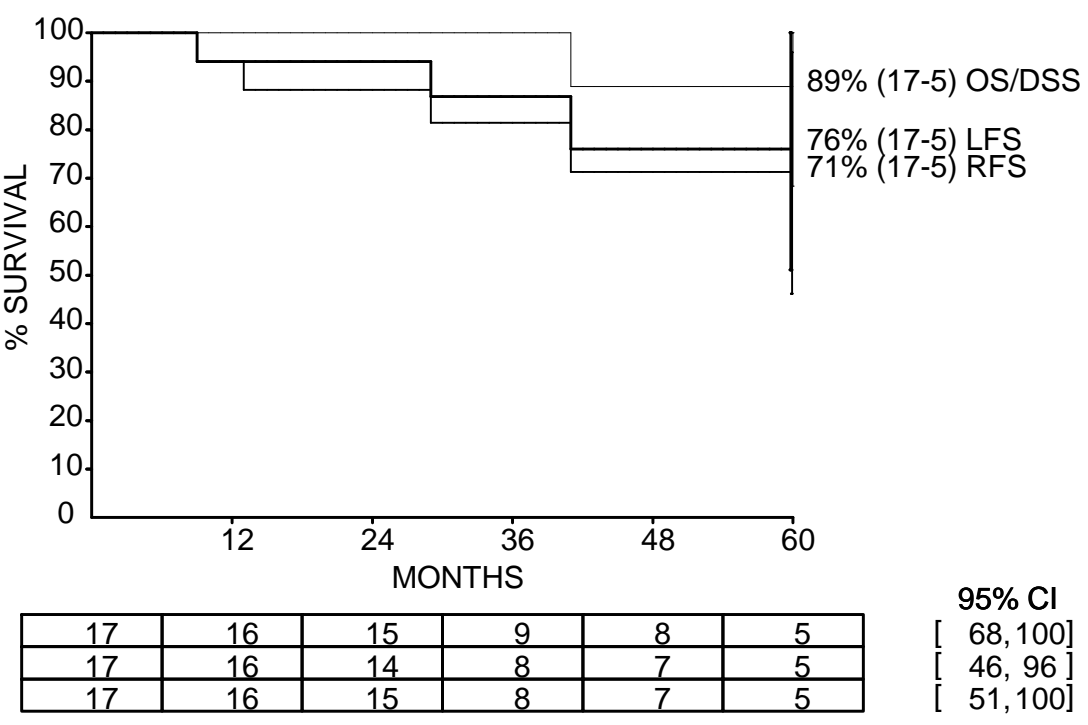

Figure 1: Treatment Outcome at 5 Years.

OS: Overall Survival; LFS: Laryngectomy Free Survival; DSS: Disease Specific Survival; RFS: Relapse Free Survival

Prior to treatment, all patients had $\geq$ grade 2 voice changes $(88 \%$ with grade $2 ; 12 \%$ with grade 3$)$. At 12 months (+/- 2 months) after completing CRT, the prevalence of $\geq$ grade 2 voice changes decreased to $44 \%$ (all grade 2 , no grade 3 toxicity). The prevalence of that at the date last seen was 33\% (all grade 2, no grade 3 toxicity). The median interval from completion of concurrent CRT to the date last seen was 42 months (range: 13-106 
months). No patient had $\geq$ grade 2 dysphagia prior to treatment while at 12 months $(+/-2$ months) after completing CRT, one patient had grade 3 dysphagia requiring tube feeding. At the date last seen, his dysphagia was downgraded to 2 because tube feeding was no longer required. The prevalence of $\geq$ grade 2 voice changes and dysphagia at 3 different time points were shown in Figures 2 and 3 .

\section{Voice Changes}

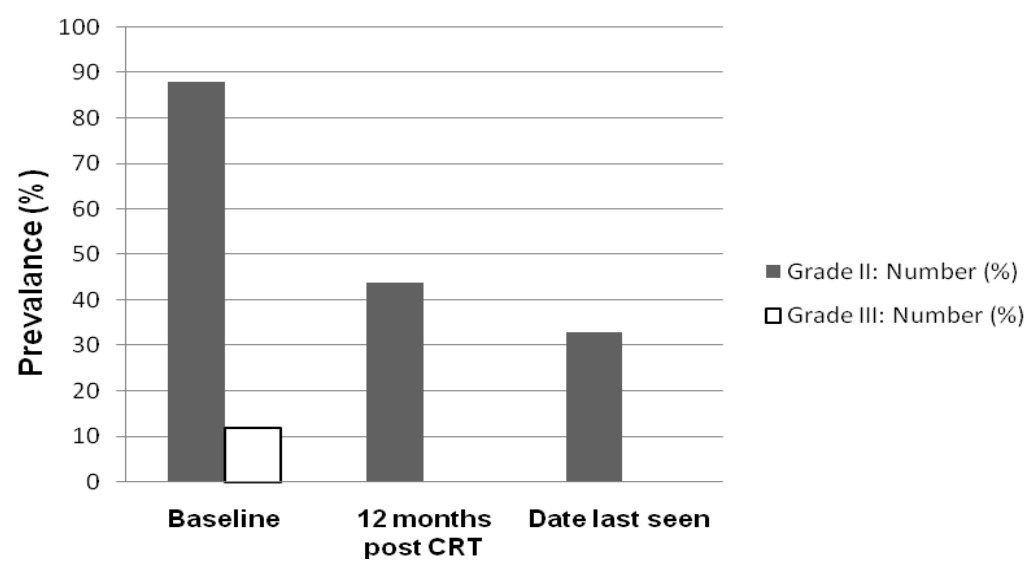

\begin{tabular}{|l|l|l|l|}
\hline Grade II: Number(\%) & $15(88 \%)$ & $7(44 \%)$ & $5(33 \%)$ \\
\hline Grade III: Number(\%) & $2(12 \%)$ & $0(0 \%)$ & $0(0 \%)$ \\
\hline Number of patients assessable & 17 & 16 & 15 \\
\hline
\end{tabular}

Figure 2. Prevalence of $\geq$ Grade 2 Voice Changes for Patients Alive and Free of Local Failure. The Median Interval from Completion of Concurrent Chemoradiation to Date Last Seen was 42 Months (Range: 13-106 Months)

\section{Dysphagia}

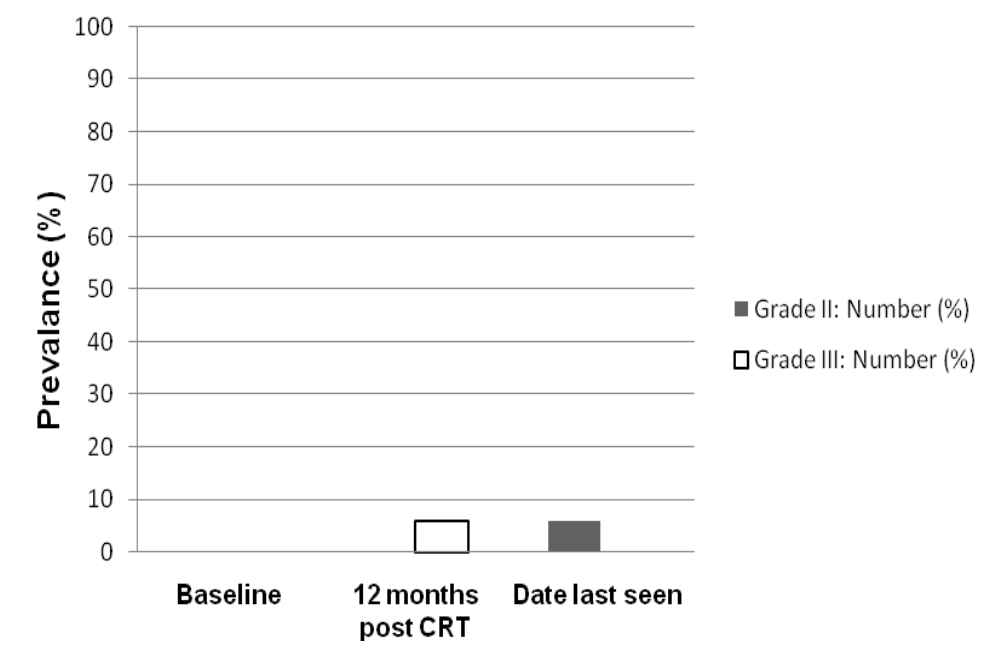

\begin{tabular}{|l|l|l|l|}
\hline Grade II: Number(\%) & $0(0 \%)$ & $0(0 \%)$ & $1(7 \%)$ \\
\hline Grade III: Number(\%) & $0(0 \%)$ & $1(6 \%)$ & $0(0 \%)$ \\
\hline Number of patients assessable & 17 & 16 & 15 \\
\hline
\end{tabular}

Figure 3. Prevalence of $\geq$ Grade 2 Dysphagia. The Median Interval from Completion of Concurrent Chemoradiation to Date Last Seen was 42 Months (Range: 13-106 Months) 


\section{Discussion}

Upfront surgery is a highly effective treatment for T3N-/+ glottic SCC but in most cases, total laryngectomy is required (Yuen A, 1984, DeSanto, 1991). While 'voice-preserving' laryngectomy may be feasible in highly-selected cases (Weinstein, 2003), there has been no prospective randomized studies to compare and contrast such procedures to total laryngectomy.

The Veteran Affairs (VA) Laryngeal Cancer study (1991) was the first randomized trial to show that patients with locoregionally advanced laryngeal SCC can be safely spared from laryngectomy. Induction chemotherapy and sequential radiotherapy were utilized in the design of VA study and those with significant larynx destruction (bulky T4 disease) were excluded. Forastiere et al. (Forastiere AA, 2003) reported the outcome of RTOG 91-11 study which compared three larynx preservation approaches (induction chemotherapy followed by radiotherapy, concurrent CRT, and radiotherapy alone) for locally and/or regionally advanced larynx SCC. The RTOG 91-11 study showed that CRT had the highest rate of laryngeal preservation when compared to the other two approaches. These two landmark randomised controlled trials enrolled patients with T3 and T4 supraglottic and glottic SCC. There were 58 patients with T3 glottic SCC in the RTOG 91-11 trial but the outcome of that particular group was not sub-analysed.

Apart from the two large randomized control studies which included T3 and T4 laryngeal SCC, several published uncontrolled series specifically examined the rate of larynx preservation for T3 glottic SCC. Bryant et al (Bryant GP, 1995) reported the treatment outcome from Queensland Radium over a 29 year period. The $5 \mathrm{Yr}$ DSS for upfront RT alone +/- salvage laryngectomy was 50\% while the 5 year relapse-free survival was $33 \%$ for patients with T3 glottic SCC. In that study, over $50 \%$ of patients treated with initial RT alone retained their larynx. Sessions et al (Sessions DG, 2002) reported close to $70 \%$ rate of larynx preservation with RT alone, supported by Lundgren et al (Lundgren JAV 1988) and Mendenhall et al (Mendenhall WM, 1992) where the rate of 5 years larynx preservation were $59 \%$ and $63 \%$ respectively (Table 1 ).

\section{Table 1. Larynx Preservation for T3N+/-MO Glottic SCC: Comparison of Current Study to Other Published Series}

\begin{tabular}{|l|l|l|l|}
\hline & Number of patients & Larynx preserving treatment & Rate of Larynx preservation \\
\hline Bryant et al. & 55 & Radiotherapy & Just over 50\% at 5 years \\
\hline Lundgren et al. & 141 & Radiotherapy & $59 \%$ at 5 years \\
\hline Mendenhall et al. & 73 & Radiotherapy & $63 \%$ at 5 years \\
\hline \multirow{2}{*}{ Sessions et al. } & 29 & Radiotherapy & $69.5 \%$ at 5 years \\
\cline { 2 - 4 } & 22 & Conservative Surgery & $54.5 \%$ at 5 years \\
\hline Spector et al. & 26 & $\begin{array}{l}\text { Radiotherapy or chemoradiation or } \\
\text { conservative surgery +/- Radiotherapy }\end{array}$ & $88.5 \%$ at 3 years \\
\hline Current study & 17 & Chemoradiation & $87 \%$ at 5 years \\
\hline
\end{tabular}

In another study, Spector et al (Spector JG, $2006)$ reported the outcome of 26 patients with T3 glottic SCC treated with various larynx-preservation approaches. In that study, 6 patients were treated with RT alone, one treated with concurrent CRT, while 19 received conservative surgery $+/$ - adjuvant RT. The overall rate of larynx preservation was $88.5 \%$ at 3 years. It is important to note that none of these uncontrolled studies specifically investigated the use of concurrent CRT (except one patient by Spector et al who was free of disease at the last follow up). It would seem reasonable to suggest that our series compared favorably to other published uncontrolled series by the addition of concurrent Cisplatin-based chemotherapy. We would like to admit that the findings from our audit is by no means ground breaking, but it will hopefully provide further evidence to what is generally 
considered as the standard management for T3N+/-M0 glottic SCC.

Recent publication of retrospective data from the National Cancer Data Base by Hoffman et al. sparked the debate on larynx preservation suggesting that non-surgical management may worsen the overall survival for patients in the United States with advanced laryngeal malignancy.(Hoffman et al., 2006) This lead to the call for a reevaluation of treatment for advanced laryngeal cancer by Dr. Kerry Olsen.(Olsen, 2010) Olsen et al. indicated that the decline in overall survival for T3 laryngeal cancer (including T3 glottic SCC) was secondary to the increasing use of non-surgical treatment (radiotherapy +/- chemotherapy). Olsen felt that laryngeal conservation surgery (partial vertical laryngectomies, supraglottic laryngectomies, supracricoid operations, and transoral laser or robitic surgery) for advanced laryngeal cancer is under utilized.

In Hoffman's paper, analysis was performed for T3N0M0 laryngeal SCC. Although a statistically significant difference was detected in 5 year survival between patients treated surgically (+/- irradiation) and those treated with RT (+/- chemotherapy), direct analysis between the observed 5 year survival of $59.3 \%$ for chemoradiation was not significantly different from the $63.3 \%$ of surgery $(p=0.503)$. Similarly, identical 5 year survival (65.6\%) was noted after initial treatment with chemoradiation and surgery combined with RT for patients with T3N0M0 glottic SCC.(Hoffman et al., 2006) Thus the notion that larynx preservation with non-surgical management for T3 laryngeal carcinoma (including glottic SCC) jeopardized survival is not supported as per the commentary by Forastiere. (Forastiere, 2010)

The use of laryngeal-preserving surgery for T3/T4 larynx SCC is highly selective. Not many surgeons may have the experience or expertise to perform these procedures due to the decrease in resident training and experience in laryngeal conservation surgery as correctly suggested by Olsen.(Olsen, 2010) That was certainly the case in our centre as our head and neck surgeons did not feel comfortable with such approach and also due to the lack of level two evidence to support that laryngeal-preserving surgery can achieve the same level of oncological outcome as that of total laryngectomy.

The voice quality would have been better assessed by the speech pathologists. It is not currently our standard practice to request speech pathology assessment prior to and after CRT for laryngeal SCC, unless there is a concern for aspiration. Furthermore, many of these patients came from rural areas in Queensland, where speech pathology follow up is difficult to arrange for logistical reasons. Using CTC criteria, our study detected relatively low rate of dysphagia. The prevalence of $\geq$ grade 2 dysphagia was $6 \%$ at 12 months from completion of concurrent CRT and at date last seen. In terms of voice, while $33 \%$ of our cohort had grade 2 voice changes at date last seen, all of those alive and not had a laryngectomy were able to get by verbal communication on the telephone. We also noted that voice improves with time in patients who were free of local failure, and not had laryngectomy as illustrated by the reducing prevalence of grade two voice changes over time.

We acknowledge that this study is of retrospective nature and the sample size is small. Nevertheless, it demonstrated satisfactory local control and acceptable overall survival with concurrent CRT for this subset of patients with $\mathrm{T} 3 \mathrm{~N}+/-\mathrm{M} 0$ glottic SCC. The rate of long term dysphagia associated with such treatment was low. Knab et al (Knab BR, 2008) reported a series of 32 patients with T4 laryngeal disease (9\% confined to true glottis) treated by induction chemotherapy followed by concurrent CRT using twice daily RT regimen. Some of these patients had large T4 disease defined as tumour penetrating through thyroid cartilage and/or more than $1 \mathrm{~cm}$ invasion into the base of tongue. Local control at 4 years was $83 \%$ while the rate of intact larynx at 4 years was $86 \%$. At date last seen, $15 \%$ of the cohort was dependant on feeding tube. The majority of patients with T4N+/-M0 SCC of the glottis would be treated with total laryngectomy in our institution. While our results for $\mathrm{T} 3 \mathrm{~N}+/-\mathrm{M} 0$ glottic SCC treated with concurrent CRT is encouraging, it would 
seem reasonable to start offering concurrent CRT to some of the T4 patients when meaningful voice preservation can be achieved. A study by Levendag et al. demonstrated a dose-dysphagia relationship in head and neck radiotherapy.(Levendag PC 2007) The dose received by the pharyngeal constrictor muscle was found to correlate to the incidence of long term swallowing difficulties. As the pharyngeal constrictor muscle is located posterior to the glottic larynx, treating some of the T4 disease where tumour infiltrates anteriorly to structures such as thyroid cartilage is not anticipated to increase the dose to the constrictor muscles. Conversely, posterior extension of the glottic tumour to the posterior pharyngeal wall or hypopharynx is likely to result in significant dose increment to the pharyngeal constrictor muscles, potentiating higher incidence of long term dysphagia.

\section{Conclusions}

Concurrent CRT offered high rate of larynx preservation with low incidence of persistent dysphagia in patients with $\mathrm{T} 3 \mathrm{~N}+/-\mathrm{M} 0$ glottic SCC.

\section{Competing Interests}

The authors declare that they have no competing interests

\section{Authors' Contributions}

CL participated in the study design, coordinated the study and write the manuscript.

ES participated in the data collection and drafting of the manuscript.

JK participated in the design of the study, acquisition and interpretation of the data.

\section{Acknowledgements}

The authors would like to thank Mr. Lee Tripcony for his contribution in statistical analysis.

\section{References}

Bryant, G. P., Poulsen. M. G. , Tripcony, L. \& Dickie, G. J. (1995). "Treatment Decisions in T3N0M0 Glottic Carcinoma," Int. J. Radiation Oncology Biol. Phys, 31, 285-293.

Desanto, L. W. (1991). 'T3 Glottic Cancer:Options and Consequences of the Options,' Laryngoscope, 94, 1311-1315.

Forastiere, A. A. (2010). "Larynx Preservation and Survival Trends: Should There be Concern?," Head Neck, 32, 14-7.

Forastiere, A. A., Goepfert, H., Maor, M. et al. (2003). "Concurrent Chemotherapy and Radiotherapy for Organ Preservation in Advanced Laryngeal Cancer," N Eng J Med, 349, 2091-2098.

Hoffman, H. T., Porter, K., Karnell, L. H., Cooper, J. S., Weber, R. S., Langer, C. J., Ang, K.- K., Gay, G., Stewart, A. \& Robinson, R. A. (2006). "Laryngeal Cancer in the United States: Changes in Demographics, Patterns of Care, and Survival," Laryngoscope, 116, 1-13.

Insitutute, N. C. (2006). 'Common Terminology Criteria for Adverse Events v3.0 (CTCAE),'

Knab, B. R., Sama, J. K., Solanki, A. et al. (2008). "Functional Organ Preservation with Definitive Chemoradiotherapy for T4 Laryngeal Squamous Cell Carcinoma," Annals of Oncology, 19, 1650-1654.

Levendag, P. C., Teguh, D. N., Voet, P. et al. (2007). "Dysphagia Disorders in Patients with Cancer of the Oropharynx are Significantly Affected by the Radiation Therapy Dose to the Superior and Middle Constrictor Muscle: A Dose-Effect Relationship," Radiotherapy and Oncology, 83, 64-73.

Lundgren, J. A. V. , Gilbert, R. W. , Nostrand, A. W. P., Harwood, A. R., Keane, T. J. \& Briant, T. D. (1988). "T3N0M0 Glottic Carcinoma-A Failure Analysis," Clin Otolaryngol 13, 455-465. 
Mendenhall, W. M., Parsons, J. T., Stringer, S. P., Cassisi, N. J. \& Million, R. R. (1992). “Stage T3 Squamous Cell. Carcinoma of the Glottic Larynx: A Comparison of Laryngectomy and Irradiation," Int J Radiat Oncol Biol Phy, 23, 725-732.

Olsen, K. D. (2010). "Reexamining the Treatment of Advanced Laryngeal Cancer," Head Neck, 32, 1-7.

Sessions, D. G., Lenox, J., Spector, G., Newland, D. , Simpson, J., Haughey, B. H. \& Chao, K. S. (2002). "Management of T3N0M0 Glottic Carcinoma: Therapeutic Outcomes," The Laryngoscope, 112, 1281-1288.

Spector, J. G., Sessions, D. J., Lenox, J. \& Simpson, J. (2006). "Management of T3N1 Glottic Carcinoma:Therapeutic Outcomes," Laryngoscope, 116, 106-110.

The Department of Veterans Affairs Laryngeal Cancer Study Group. (1991). "Induction Chemotherapy Plus Radiation Compared with Surgery Plus Radiation in Patients with Advanced Laryngeal Cancer," N Engl J Med, 324, 1685-90.

Weinstein, G. S. (2003). “Organ Preservation Surgery for Laryngeal Cancer: The Evolving Role of the Surgeon in the Multidisciplinary Head and Neck Cancer Team," Oper Tech Otolaryngol Head Neck Surg, 13, 1-2.

Yuen, A., Mdina, J. E., Goepfert, H. \& Fletcher, G. (1984). "Management of Stage T3 and T4 Glottic Carcinoma," Am J Surg, 148, 467-472. 